\title{
First simultaneous multi-wavelength observations of the black hole candidate IGR J17091-3624
}

\section{ATCA, INTEGRAL, Swift, and RXTE views of the 2011 outburst ${ }^{\star}$}

\author{
J. Rodriguez ${ }^{1}$, S. Corbel ${ }^{1}$, I. Caballero ${ }^{1}$, J. A. Tomsick ${ }^{2}$, T. Tzioumis ${ }^{3}$, A. Paizis ${ }^{4}$, M. Cadolle Bel ${ }^{5}$, and E. Kuulkers ${ }^{5}$ \\ ${ }^{1}$ Laboratoire AIM, CEA/IRFU, CNRS/INSU, Université Paris Diderot, CEA DSM/IRFU/SAp, 91191 Gif-sur-Yvette, France \\ e-mail: jrodriguez@cea.fr \\ 2 Space Sciences Laboratory, 7 Gauss Way, University of California, Berkeley, CA 94720-7450, USA \\ 3 Australia Telescope National Facility, CSIRO, PO Box 76, Epping NSW 1710, Australia \\ ${ }^{4}$ Istituto Nazionale di Astrofisica, INAF-IASF, via Bassini 15, 20133 Milano, Italy \\ 5 ESAC, ISOC, Villañueva de la Cañada, Madrid, Spain
}

Received 17 June 2011 / Accepted 31 July 2011

ABSTRACT

\begin{abstract}
We present the results of the first four (quasi-)simultaneous radio (ATCA), X-ray (Swift, RXTE), and $\gamma$-ray (INTEGRAL) observations of the black hole candidate IGR J17091-3624, performed in February and March 2011. The X-ray analysis shows that the source was in the hard state, and then it transited to a soft intermediate state. We study the correlated radio/X-ray behaviour of this source for the first time. The radio counterpart to IGR J17091-3624 was detected during all four observations with the ATCA. In the hard state, the radio spectrum is typical of optically thick synchrotron emission from a self-absorbed compact jet. In the soft intermediate state, the detection of optically thin synchrotron emission is probably due to a discrete ejection event associated with the state transition. The position of IGR J17091-3624 in the radio versus X-ray luminosity diagram (aka fundamental plane) is compatible with that of the other black hole sources for distances greater than $11 \mathrm{kpc}$. IGR J17091-3624 also appears as a new member of the few sources that show a strong quenching of radio emission after the state transition. Using the estimated luminosity at the spectral transition from the hard state, and for a typical mass of $10 M_{\odot}$, we estimate a distance to the source between $\sim 11$ and $\sim 17 \mathrm{kpc}$, compatible with the radio behaviour of the source.
\end{abstract}

Key words. accretion, accretion disks - X-rays: binaries - radio continuum: stars - stars: individual: IGR J1709-8722;3624

\section{Introduction}

Most of the $\sim 40$ Galactic black hole binaries (BHB) are transient sources that are detected in X-rays during bright, months-to-year long outbursts. During this period, their luminosity varies by several orders of magnitude. At the same time, the broad band ( 0.1-200 keV) X-ray spectra show pivoting from the canonical hard state (HS) to the canonical soft state (SS), through intermediate flavours of these last two. These states also have clear signatures in the temporal domain $(0.1-1000 \mathrm{~Hz})$, with the appearance of different types (dubbed A, B, C) of quasi periodic oscillations (QPO) in the HS, hard intermediate (HIMS), and soft intermediate (SIMS) states (e.g. Remillard \& McClintock 2006; Homan et al. 2005; Remillard et al. 2002).

BHBs are also known to produce strong radio emission, either from a powerful compact jet in the HS or from relativistic discrete ejections at the transition from the HIMS to the SIMS (e.g., Fender 2006). In the HS, a strong correlation between the X-ray and radio luminosity has been found by Corbel et al. (2003) who point out a very strong coupling between the compact jets and the inner accretion flow. For Cyg X-1, this jet has been detected above $\sim 500 \mathrm{keV}$ (Laurent et al. 2011). The $L_{\text {Radio }}=L_{\mathrm{X}}^{0.6}$ relation between the radio and X-ray luminosities is thought to be universal (aka the "standard" pattern or GX 339-4 like behaviour), although it is based on only eight Galactic sources (among which GX 339-4 and Cyg X-1 show a

\footnotetext{
* Table 4 is available in electronic form at http: //www . aanda. org
}

large quenching of the radio emission in the SS; Corbel et al. 2003; Fender et al. 2010). Seven sources have recently been shown to define a new track in the radio versus X-ray luminosity diagram by following the relation $L_{\text {Radio }}=L_{\mathrm{X}}^{1.4}$ (hereafter the H1743-322 like behaviour; Fender et al. 2010; Coriat et al. 2011). The total number of BHBs simultaneously followed in a multi-wavelength way is, however, still low. It is, thus, not clear which of the two types of behaviour (GX 339-4 or H1743-322like) is the most common, or what leads to these differences. Multi-wavelength observations of any new active target, over the widest possible range of luminosities are, therefore, important for better understanding the jet behaviour and assessing the links between the accretion and ejection processes. To this end, we conducted Australian Telescope Compact Array (ATCA) and Rossi X-ray Timing Explorer (RXTE) observations as soon as IGR J17091-3624 was seen to enter a new outburst in 2011.

IGR J17091-3624 was discovered with the INTErnational Gamma-Ray Astrophysics Laboratory (INTEGRAL) on April 14, 2003 while entering outburst (Kuulkers et al. 2003). Observations with other facilities (e.g. RXTE, Swift) permitted it to be classified as a low-mass X-ray binary, which was probably hosting a black hole (e.g. Lutovinov et al. 2005; Capitanio et al. 2009). The renewal of activity in late January 2011 (Krimm et al. 2011; Krimm \& Kennea 2011) prompted several observing campaigns on the source. It was, in particular, found that IGR J17091-3624 has similarities with GRS 1915+105, since it showed types of X-ray variability reminiscent of those 
A\&A 533, L4 (2011)

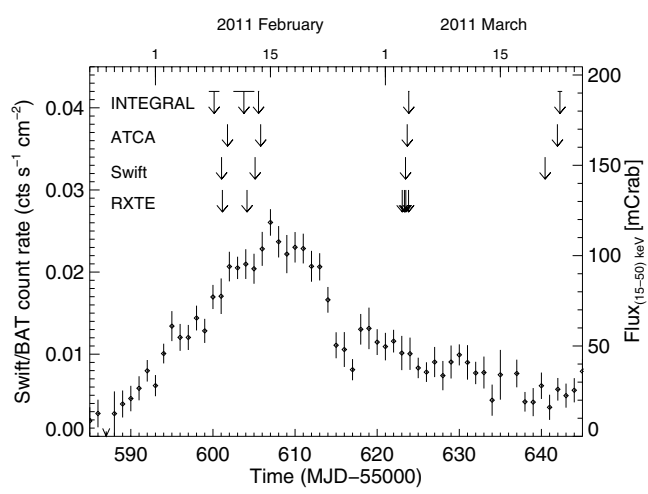

Fig. 1. 15-50 keV Swift/BAT lightcurve of IGR J17091-3624. The vertical arrows represent the (mid) times of the pointed observations. The width of the arrow's top is the length of the observation (visible only for INTEGRAL).

of the so-called " $\rho$-heartbeat" and " $\beta$ " classes seen in GRS 1915+105 (Altamirano et al. 2011a,b). The specifics of the IGR J17091-3624's X-ray behaviour further justify a (simultaneous) multi-wavelength study, to see, in particular, how it compares to the other $\sim 17 \mathrm{BHBs}$ with known radio-X-ray behaviour.

Early in the outburst, we reported the presence of radio emission compatible with a compact jet in the HS (Corbel et al. 2011) and a $\sim 0.1 \mathrm{~Hz}$ QPO at the same time (Rodriguez et al. 2011). Here, we present an in-depth analysis of four (quasi) simultaneous radio-X- $\gamma$-ray observations. To complement the ATCA and RXTE data, we use INTEGRAL and Swift observations to enhance the spectral coverage.

\section{Observations and data reduction}

Figure 1 shows the $15-50 \mathrm{keV}$ daily averaged Swift/BAT light curve of IGR J17091-3624, with the various observations discussed in this paper. The journal of these observations can be found in Table 4 . The observations are, hereafter, ordered and labelled according to the instrument used (A stands for ATCA, I for INTEGRAL, S for Swift, and R for RXTE). The ATCA observations were made in two frequency bands $(5.5$ and $9 \mathrm{GHz})$ simultaneously, with the upgraded Compact Array Broadband Backend (Wilson et al. 2011). The amplitude and band-pass calibrator was PKS 1934-638, and the antenna's gain and phase calibration, as well as the polarisation leakage, were derived from regular observations of the nearby $\left(\sim 3.2^{\circ}\right.$ away $)$ calibrator PMN 1714-336. See, e.g., Corbel et al. (2004) for more details on the standard data reduction.

The RXTE and Swift data were reduced with HEASOFT ${ }^{1}$ v6. 10, and the INTEGRAL data with OSA v9. $0^{2}$. The X-ray spectral fitting software package XSPEC $12.6 .0 \mathrm{q}$ was used to fit the energy and power density spectra (PDS) after converting the latter into XSPEC readable files.

The Swift/XRT level 2 cleaned event files were obtained from window timing (WT) mode data with xrtpipeline keeping only the grade 0 events. We then extracted the spectra from a $40 \times 10$ pixel region centred on the best source position. The background was estimated from a region of the same size at an off-axis position. The ancillary response file (arf) was estimated with xrtmkarf, and the last version (v. 12) of the WT redistribution matrix file (rmf) was used in the spectral fits.

\footnotetext{
1 http://heasarc.gsfc.nasa.gov/docs/software/lheasoft/

${ }^{2}$ http://www.isdc.unige.ch/integral/analysis\#Software
}

Table 1. Radio fluxes of IGR J17091-3624 during the four ATCA observations.

\begin{tabular}{cccc}
\hline \hline Obs. & $\begin{array}{c}F_{5.5 \mathrm{GHz}} \\
(\mathrm{mJy})\end{array}$ & $\begin{array}{c}F_{9 \mathrm{GHz}} \\
(\mathrm{mJy})\end{array}$ & $\alpha$ \\
\hline $\mathrm{A} 1$ & $1.40 \pm 0.05$ & $1.24 \pm 0.06$ & $-0.25 \pm 0.12$ \\
$\mathrm{~A} 2$ & $1.53 \pm 0.10$ & $1.57 \pm 0.10$ & $+0.05 \pm 0.19$ \\
$\mathrm{~A} 3$ & $2.41 \pm 0.10$ & $1.13 \pm 0.10$ & $-1.54 \pm 0.20$ \\
$\mathrm{~A} 4$ & $0.17 \pm 0.05$ & $<0.08$ & \\
\hline
\end{tabular}

Notes. Errors are at the $1 \sigma$ level.

The INTEGRAL/IBIS/ISGRI source spectra were obtained in a standard way (e.g. Rodriguez et al. 2008a). We used the latest version of the rmf and arf files available from the INTEGRAL instrument calibration tree provided with OSA 9.0. In two observations, the source is also within the field of view (fov) of the JEM-X monitors, and we extracted 16 channels spectra from JEM-X unit 2.

The Z source GX $349+2$ is at $\sim 41^{\prime}$ from IGR J17091-3624. The RXTE/PCA data are thus contaminated by the emission from this bright source at a level that is very difficult to estimate given the lack of strictly simultaneous data. The RXTE/PCA data were therefore only considered for timing analysis (e.g. Rodriguez et al. 2011). Two to $60 \mathrm{keV}$ PDS were extracted from Good Xenon mode date with POWSPEC v1.0 over the $0.0078125-500 \mathrm{~Hz}$ range. The fits of these PDSs were restricted to the frequency range $0.0078125-30 \mathrm{~Hz}$.

\section{Results}

\subsection{Radio observations}

The analysis of the ATCA data shows there is a single radio source within the X-ray error circle, at a location of $(\alpha, \delta)=$ $17^{\mathrm{h}} 09^{\mathrm{m}} 07^{\mathrm{s}} .61,-36^{\circ} 24^{\prime} 25^{\prime \prime} .7(\mathrm{~J} 2000,1 \sigma$ positional uncertainty of $\left.0.1^{\prime \prime}\right)$. This position is also consistent with the position of the suggested optical/NIR counterpart (Torres et al. 2011). The measured radio fluxes and spectral indices $\alpha$ (defined by $\mathrm{F}(v) \propto v^{\alpha}$ ) are reported in Table 1.

\section{2. $X$ and $\gamma$-ray spectral analysis}

The Swift and INTEGRAL spectra S1+I1, S2+I2, S3+I3, and $\mathrm{S} 4+\mathrm{I} 4$ were fitted simultaneously, and we retained the energy channels $0.7-7.5 \mathrm{keV}$ for XRT, 20-300 keV for ISGRI, and, in $\mathrm{S} 1+\mathrm{I} 1$ and $\mathrm{I1}^{\prime}$, the 4-20 keV channels for JEM-X2. The bestfit models consist of an absorbed ${ }^{3}$ power law with a high energy cut-off (tbabs*cutoffpl in XSPEC, hereafter CPL) for spectra $\mathrm{S} 1+\mathrm{I} 1, \mathrm{I} 1^{\prime}$, and $\mathrm{S} 2+\mathrm{I} 2$. A disc component is required in $\mathrm{S} 3+\mathrm{I} 3$ and $\mathrm{S} 4+\mathrm{I} 4$, while the cut-off is not needed any more (tbabs*(diskbb+powerlaw), hereafter DPL). A normalisation constant was also included and frozen to 1 for the XRT spectra and left free to vary for the other instruments, except for the I1' fits, where it is frozen to 1 for ISGRI. The best-fit parameters we obtained are reported in Table 2, and the " $v-F_{v}$ " spectra of $\mathrm{S} 1+\mathrm{I} 1$ and $\mathrm{S} 3+\mathrm{I} 3$ are represented in Fig. 2.

In XRBs, a cut-off power-law spectrum is usually interpreted as the signature of inverse Comptonisation of soft seed photons by a thermalised (i.e., with velocities following a Maxwellian distribution) population of electrons. A thermal Comptonisation model (comptt Titarchuk 1994) provided a good fit to S1+I1, I1', and S2+I2 (ThC in Table 2). We assumed

\footnotetext{
3 Abundances fixed to those from Wilms et al. (2000).
} 
Table 2. Results of the spectral fits to the joint Swift/XRT and INTEGRAL/ISGRI (and JEM-X in I1 and I1') spectra.

\begin{tabular}{|c|c|c|c|c|c|c|c|c|c|}
\hline \multirow[t]{2}{*}{ Spectra } & \multirow[t]{2}{*}{ Model } & \multirow{2}{*}{$\begin{array}{c}N_{\mathrm{H}} \\
\left(\times 10^{22} \mathrm{~cm}^{-2}\right)\end{array}$} & \multirow{2}{*}{$\begin{array}{c}k T_{b b} / k T_{\text {inj }} \\
(\mathrm{keV})\end{array}$} & \multirow[t]{2}{*}{$\Gamma / \tau$} & \multirow{2}{*}{$\begin{array}{c}E_{\mathrm{cut}} / k T_{e} \\
(\mathrm{keV})\end{array}$} & \multirow{2}{*}{$\begin{array}{c}\chi_{v}^{2} \\
\text { (d.o.f.) }\end{array}$} & \multicolumn{3}{|c|}{ Unabs. Flux ${ }^{\star}$} \\
\hline & & & & & & & $0.5-10 \mathrm{keV}$ & $3-9 \mathrm{keV}$ & $20-200 \mathrm{keV}$ \\
\hline \multirow[t]{2}{*}{$\bar{S} 1+\mathrm{I} 1$} & CPL & $1.08 \pm 0.08$ & & $1.40 \pm 0.07$ & $100_{-13}^{+17}$ & $0.90(89)$ & 8.4 & 4.5 & 20.0 \\
\hline & ThC & $1.14_{-0.05}^{+0.06}$ & 0.2 (frozen) & $0.8 \pm 0.3$ & $85_{-22}^{+43}$ & $1.00(89)$ & 8.5 & 4.3 & 17.2 \\
\hline \multirow[t]{2}{*}{$\mathrm{I} 1^{\prime}$} & CPL & 1.1 frozen & & $1.49 \pm 0.09$ & $91_{-13}^{-172}$ & $1.0(34)$ & NA & 6.5 & 22.4 \\
\hline & $\mathrm{ThC}$ & 1.1 frozen & 0.2 (frozen) & $0.8 \pm 0.2$ & $74_{-10}^{+22}$ & $1.0(34)$ & NA & 7.2 & 22.3 \\
\hline \multirow[t]{2}{*}{$\mathrm{S} 2+\mathrm{I} 2$} & CPL & $1.15 \pm 0.06$ & & $1.54 \pm 0.05$ & $107_{-32}^{+61}$ & $1.1(137)$ & 12.1 & 6.0 & 20.8 \\
\hline & $\mathrm{ThC}$ & $1.13 \pm 0.05$ & 0.2 (frozen) & $2.1_{-1.5}^{+0.5}$ & $27_{-7}^{+74}$ & $1.2(137)$ & 11.7 & 6.0 & 25.9 \\
\hline $\mathrm{S} 3+\mathrm{I} 3$ & DPL & $1.5 \pm 0.1$ & $1.2 \pm 0.2$ & $2.3 \pm 0.3$ & & $1.3(128)$ & 38.2 & 11.4 & 7.3 \\
\hline $\mathrm{S} 4+\mathrm{I} 4^{\circ}$ & DPL & $1.16 \pm 0.05$ & $1.29_{-0.03}^{+0.01}$ & $2.1_{-0.3}^{+0.1}$ & & $1.2(409)$ & 31.6 & 12.4 & 2.1 \\
\hline
\end{tabular}

Notes. Errors are at the $90 \%$ level. ${ }^{(\star)}$ In units of $10^{-10} \mathrm{erg} \mathrm{cm}^{-2} \mathrm{~s}^{-1}$. Fluxes are normalised to Swift/XRT, except I1' normalised to ISGRI. (०) Observations made more than a day apart. Norm. constant is high and poorly constrained $(\gtrsim 2)$.

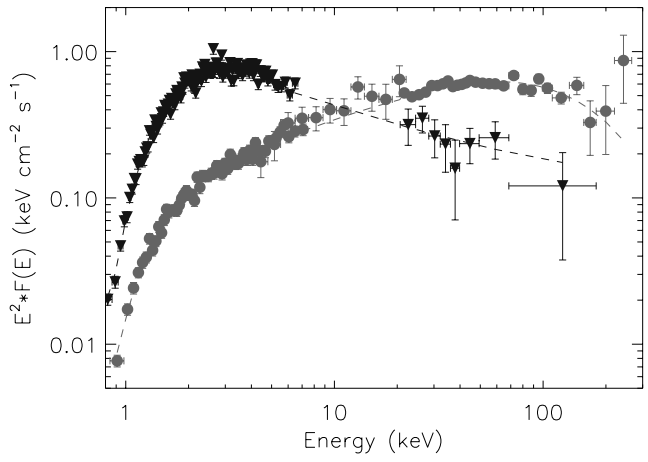

Fig. 2. Spectra of obs. S1+I1 (XRT/JEM-X 2/ISGRI, grey filled circles) and $\mathrm{S} 3+\mathrm{I} 3$ (XRT/ISGRI, dark triangles). The dashed lines represent the best-fit models.

a disc geometry in XSPEC and fixed the temperature of the seed photons. This model also permits avoiding the divergence of the power-law flux towards low energy since Comptonisation significantly contributes above $3 \times k T_{\text {inj }}$ and decreases quickly below.

\subsection{X-ray timing analysis}

None of the RXTE observations shows the " $\rho$-heartbeat" type of variability. Since GX 349+2 contaminates significantly Obs. R1 and R2 (Sect. 2), we do not discuss the level of continuum variability since we cannot attribute it to IGR J17091-3624 with certainty. The IGR J17091-3624 PDSs, however, show QPOs that are not associated with GX 349+2 (Rodriguez et al. 2011; Shaposhnikov 2011; Pahari et al. 2011). To obtain their "true" amplitudes during Obs. R1 and R2, we have estimated the net count rate of IGR J17091-3624 in the RXTE/PCA by using our spectral results (Table 2) and simulated a PCA spectrum in XSPEC. The amplitude of the QPO is then $A_{\text {net }}=A_{\text {raw }} \times \frac{C R_{\mathrm{PCA}}}{C R_{\text {net }}}$, where $A_{\text {raw }}$ is the rms amplitude of the QPO obtained from the fit to the PDS, $C R_{\text {net }}$ the simulated count rate of IGR J17091-3624, and $C R_{\mathrm{PCA}}$ the observed PCA rate. No correction was applied to R3a,b,c,d since GX 349+2 was out of the PCA fov. The QPO parameters obtained from the analysis are reported in Table 3, and two representative PDSs are plotted in Fig. 3. These QPOs can be classified as type C QPOs, except maybe those found in observations R3 that could be transitory between $\mathrm{C}$ and $\mathrm{B}$.

\section{Discussion}

In the first two observations, the spectral parameters (Table 2) and presence of type C QPOs are typical of a BH in the HS (e.g. Remillard \& McClintock 2006; Homan et al. 2005). During the
Table 3. Parameters of the QPOs seen during Obs. R1, R2, and R3.

\begin{tabular}{lccc}
\hline \hline PDS & $\begin{array}{c}v_{\mathrm{QPO}} \\
(\mathrm{Hz})\end{array}$ & $\begin{array}{c}Q \\
(=v / F W H M)\end{array}$ & $\begin{array}{c}A \\
(\% \mathrm{rms})\end{array}$ \\
\hline R1 & $0.081_{-0.004}^{+0.005}$ & $4.3 \pm 2.2$ & $12.3_{-1.7}^{+1.8}$ \\
R2 & $0.100_{-0.008}^{+0.005}$ & $4.2 \pm 1.5$ & $10.1_{-1.6}^{+1.2}$ \\
R3a & $4.57 \pm 0.08$ & $4.2 \pm 1.1$ & $8.8 \pm 1.2$ \\
R3b & $4.57 \pm 0.08$ & $4.5 \pm 1.3$ & $8.5_{-1.3}^{+1.1}$ \\
R3c & $4.28 \pm 0.06$ & $2.4 \pm 0.3$ & $12.5_{-0.7}^{+0.5}$ \\
R3d & $4.66 \pm 0.05$ & $5.8 \pm 1.5$ & $8.0 \pm 0.6$ \\
\hline
\end{tabular}

Notes. Errors are given at the $90 \%$ confidence level.

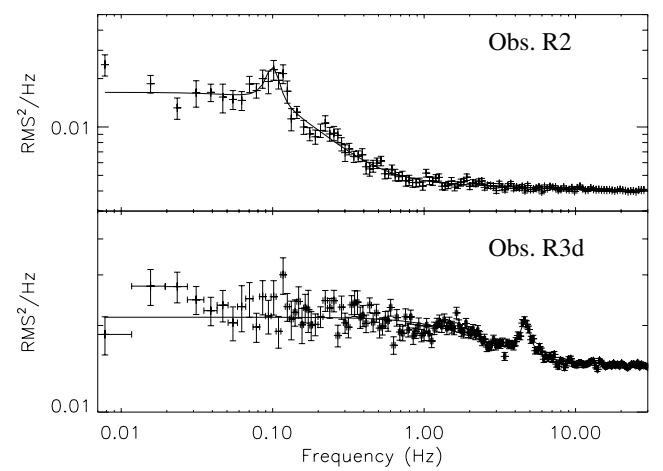

Fig. 3. PDSs of observations R2 (top panel) and R3d (bottom panel).

third observation, the source clearly is in a softer state. The transition is associated with a discrete radio ejection (see below) as often seen in BHBs (the clearest example being GRS 1915+105, e.g. Rodriguez et al. 2008a). This, and the presence of QPOs at 4-5 Hz, indicates that IGR J17091-3624 is in a SIMS at that time. No temporal (RXTE) data are available for the fourth observation. The spectra are indicative of either a SIMS or a pure SS.

The value of $N_{\mathrm{H}}$ obtained from the spectral fits is slightly higher than the $2^{\circ} \times 2^{\circ}$ weighted averaged absorption in this direction $\left(N_{\mathrm{H}}=6.0 \times 10^{21} \mathrm{~cm}^{-2}\right.$; Kalberla et al. 2005). This is consistent with a source lying at a rather large distance in the Galaxy.

The HS $\rightarrow$ SIMS state transition occurred between MJDs 55612 and 55616 (Pahari et al. 2011). Obs. 2 is the observation in the HS that is the closest to the peak of the hard X-ray outburst (Fig. 1) and to the state transition. It has a bolometric flux of $F_{\text {bol,trans }} \sim 4.1 \times 10^{-9} \mathrm{erg} \mathrm{cm}^{-2} \mathrm{~s}^{-1}$ (ThC model). Most BHBs have $L_{\text {trans, } \mathrm{HS} \rightarrow \mathrm{SS}} \gtrsim 4 \% L_{\mathrm{Edd}, 1-200 \mathrm{keV}}$, when assuming a power-law spectrum with $\Gamma=2(\text { Yu \& Yan 2009) })^{4}$.

${ }_{4}$ With exception of Cyg X-1 (HMXB) and GRO J1655-40 (discussed as an outlier by Yu \& Yan 2009). 


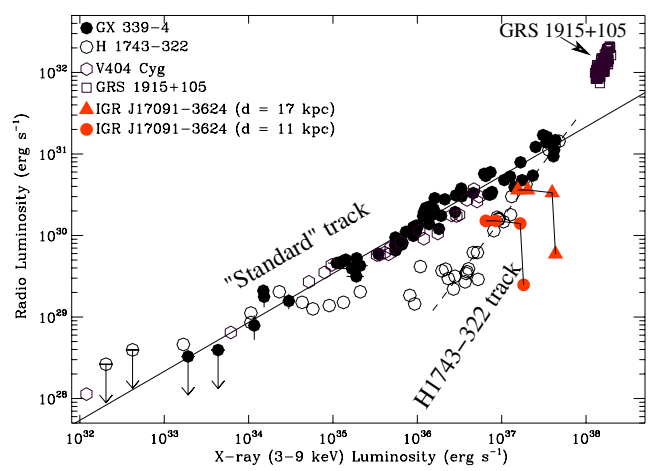

Fig. 4. Radio vs. X-ray luminosity for several BHBs.

Although this value slightly underestimates the bolometric luminosity at the transition, we take it as a "hard" lower limit on $L_{\text {trans }}$, which therefore allows us to estimate a lower limit on the distance to the source. Using $\mathrm{F}_{\text {bol,trans }}$ estimated above, we obtain a distance $d_{J 17091} \sim 11 \mathrm{kpc}$ for a BH of $\sim 10 M_{\odot}$. With $L_{\text {trans }}=10 \% L_{\text {Edd }}\left(\right.$ Esin et al. 1997) we obtain $d_{J 17091} \sim 17 \mathrm{kpc}^{5}$. Using a different method (based on the QPO frequency-photon index correlation), Pahari et al. (2011) also favour a large distance with a range of masses between $8-11.4 M_{\odot}$.

The radio spectra of observations A1 and A2 are consistent with being flat and they indicate of the presence of selfabsorbed compact jets (e.g. Corbel et al. 2004; Fender et al. 2004). Observations A3 and A4 are characteristic of optically thin synchrotron emission and can be attributed to the emission of a discrete ejection associated with the hard to soft transition. The observation of yet another BHB showing discrete ejections at the hard to soft transition probably indicates a link between transitions and (discrete) ejections potentially common to all microquasars. In XTE J1550-564 and GRS 1915+105, Rodriguez et al. (2003, 2008a,b) have suggested that the Comptonised component was the source of the ejected material. Here, the change in spectral shape and drop in the hard X-ray flux (Table 2) is compatible with this interpretation.

In Fig. 4, we plot the levels of radio and X-ray emission for IGR J17091-3624 in comparison with a sample of representative BHBs (data from Corbel et al. 2003, 2008; Coriat et al. 2011; Rushton et al. 2010) assuming the distances estimated above. The two radio points with the lowest X-ray luminosities correspond to the HS, while the other two correspond to the optically thin radio flare. At a distance of $11 \mathrm{kpc}$, the source behaviour joins the track followed by H1743-322 (Fig. 4). In this case (and at any lower distance), however, the break of the radio vs. $\mathrm{X}$-ray relation occurs at too low luminosities compared to the standard BHBs (Fender et al. 2010). At $17 \mathrm{kpc}$ the position of IGR J17091-3624 is consistent with the radio/X-ray correlation of most BHBs (within the dispersion), although we cannot assign it precisely to a specific track (i.e. the GX 339-4 or the H 1743-322 one, Fig. 4). In any cases, a large distance ( $\gtrsim 11 \mathrm{kpc}$ ) to the source is favoured by the radio-X-ray behaviour.

IGR J17091-3624 has recently been compared to GRS 1915+105 (Altamirano et al. 2011a,b; Pahari et al. 2011). However, even at the favoured distance of $17 \mathrm{kpc}$, our simultaneous radio-X-ray observations show that IGR J17091-3624 does not reach the bright levels of radio luminosity observed for GRS $1915+105$ (Fig. 4), and is more similar to standard BHBs, such as GX 339-4 or H1743-322. This would tend to indicate that the radio properties discussed here are not related to the fast flaring X-ray behaviour common to both GRS $1915+105$ and IGR J17091-3624.

Acknowledgements. We thank P. Varnière, R. Owen, and P. Curran for useful discussions. JR and SC acknowledge partial funding from the European FP7 grant agreement number ITN 215212 "Black Hole Universe". IC is supported by the CNES through CNRS. J.A.T. acknowledges partial support from NASA via INTEGRAL and Swift Guest Observer grants NNX08AX93G and NNX08AW35G. A.P. acknowledges financial contribution from the agreement ASI-INAF I/009/10/0. The Australia Telescope is funded by the Commonwealth of Australia for operation as a national facility managed by CSIRO. We acknowledge the use of the IGR Sources page (http://irfu.cea.fr/Sap/ IGR-Sources/), the use of public Swift and RXTE data obtained through the HEASARC Online Service, provided by NASA/GSFC, and observations made with INTEGRAL, an ESA project with instruments and science data centre funded by ESA member states, Poland and with the participation of Russia and the USA.

\section{References}

Altamirano, D., Belloni, T., Krimm, H., et al. 2011a, The Astronomer's Telegram, 3299, 1

Altamirano, D., Linares, M., van der Klis, M., et al. 2011b, The Astronomer's Telegram, 3225, 1

Capitanio, F., Giroletti, M., Molina, M., et al. 2009, ApJ, 690, 1621

Corbel, S., Nowak, M. A., Fender, R. P., et al. 2003, A\&A, 400, 1007

Corbel, S., Fender, R. P., Tomsick, J. A., et al. 2004, ApJ, 617, 1272

Corbel, S., Koerding, E., \& Kaaret, P. 2008, MNRAS, 389, 1697

Corbel, S., Rodriguez, J., Tzioumis, T., et al. 2011, The Astronomer's Telegram, 3167,1

Coriat, M., Corbel, S., Prat, L., et al. 2011, MNRAS, 619

Esin, A. A., McClintock, J. E., \& Narayan, R. 1997, ApJ, 489, 865

Fender, R. 2006, Jets from X-ray binaries (Compact stellar X-ray sources), 381

Fender, R. P., Belloni, T. M., \& Gallo, E. 2004, MNRAS, 355, 1105

Fender, R. P., Gallo, E., \& Russell, D. 2010, MNRAS, 406, 1425

Homan, J., Buxton, M., Markoff, S., et al. 2005, ApJ, 624, 295

Kalberla, P. M. W., Burton, W. B., Hartmann, D., et al. 2005, A\&A, 440, 775

Krimm, H. A., \& Kennea, J. A. 2011, The Astronomer's Telegram, 3148, 1

Krimm, H. A., Barthelmy, S. D., Baumgartner, W., et al. 2011, The Astronomer's Telegram, 3144, 1

Kuulkers, E., Lutovinov, A., Parmar, A., et al. 2003, The Astronomer's Telegram, 149,1

Laurent, P., Rodriguez, J., Wilms, J., et al. 2011, Science, 332, 438

Lutovinov, A., Revnivtsev, M., Molkov, S., \& Sunyaev, R. 2005, A\&A, 430, 997

Pahari, M., Yadav, J., \& Bhattacharyya, S. 2011, submitted

[arXiv: 1105.4694]

Remillard, R. A. \& McClintock, J. E. 2006, ARA\&A, 44, 49

Remillard, R. A., Sobczak, G. J., Muno, M. P., \& McClintock, J. E. 2002, ApJ, 564,962

Rodriguez, J., Corbel, S., \& Tomsick, J. A. 2003, ApJ, 595, 1032

Rodriguez, J., Hannikainen, D. C., Shaw, S. E., et al. 2008a, ApJ, 675, 1436

Rodriguez, J., Shaw, S. E., Hannikainen, D. C., et al. 2008b, ApJ, 675, 1449

Rodriguez, J., Corbel, S., Tomsick, J. A., et al. 2011, The Astronomer's Telegram, 3168, 1

Rushton, A., Spencer, R., Fender, R., \& Pooley, G. 2010, A\&A, 524, A29

Shaposhnikov, N. 2011, The Astronomer's Telegram, 3179, 1

Titarchuk, L. 1994, ApJ, 434, 570

Torres, M. A. P., Jonker, P. G., Steeghs, D., et al. 2011, The Astronomer's Telegram, 3150, 1

Wilms, J., Allen, A., \& McCray, R. 2000, ApJ, 542, 914

Wilson, W. E., Ferris, R. H., Axtens, P., et al. 2011, MNRAS, accepted [arXiv: 1105.3532]

Yu, W., \& Yan, Z. 2009, ApJ, 701, 1940

Page 5 is available in the electronic edition of the journal at http: //wWw . aanda. org

5 Fit of non simultaneous Swift/INTEGRAL observations performed on MJDs 55611-55612 leads to $F_{\text {bol,trans }} \sim 3.5 \times 10^{-9} \mathrm{erg} \mathrm{cm}^{-2} \mathrm{~s}^{-1}$. This lower value would place IGR J17091-3624 at an even farther distance. 
Table 4. Journal of the multi-wavelength observations of IGR J17091-3624 presented in this paper.

\begin{tabular}{llccc}
\hline \hline Facility & Label & $\begin{array}{c}\text { Obs. Id } \\
\text { (when available) }\end{array}$ & $\begin{array}{c}\text { MJD start } \\
\text { (d) }\end{array}$ & Good time \\
\hline ATCA & A1 & & 55601.64 & $3.1 \mathrm{~h}$ \\
& A2 & & 55605.73 & $1.9 \mathrm{~h}$ \\
& A3 & & 55623.57 & $2.2 \mathrm{~h}$ \\
& A4 & & 55641.85 & $2.4 \mathrm{~h}$ \\
RXTE & R1 & $96103-01-01-00$ & 55601.15 & $2486 \mathrm{~s}$ \\
& R2 & $96103-01-02-00$ & 55604.15 & $5668 \mathrm{~s}$ \\
& R3a & $96420-01-01-010$ & 55623.05 & $6755 \mathrm{~s}$ \\
& R3b & $96420-01-01-01$ & 55623.32 & $4695 \mathrm{~s}$ \\
Swift & R3c & $96420-01-01-020$ & 55623.49 & $16263 \mathrm{~s}$ \\
& R3d & $96420-01-01-02$ & 55623.82 & $7834 \mathrm{~s}$ \\
& S1 & 00031921005 & 55601.05 & $1457 \mathrm{~s}$ \\
& S2 & 00031921009 & 55605.13 & $2167 \mathrm{~s}$ \\
INTEGRAL & S3 & 00031921019 & 55623.45 & $686 \mathrm{~s}$ \\
& S4 & 00031921030 & 55640.45 & $2352 \mathrm{~s}$ \\
& I1 & Rev 1016 & 55599.49 & $61080 \mathrm{~s}$ \\
& I1 ${ }^{\prime} \dagger$ & Rev 1017 & 55602.49 & $131000 \mathrm{~s}$ \\
& I2 & Rev 1018 & 55605.48 & $7818 \mathrm{~s}$ \\
& I3 & Rev 1024 & 55623.77 & $6417 \mathrm{~s}$ \\
& I4 & Rev 1030 & 55641.98 & $8583 \mathrm{~s}$ \\
\hline
\end{tabular}

Notes. ${ }^{(\star)}$ INTEGRAL revolution number. ${ }^{(\dagger)} \mathrm{I} 1^{\prime}$ is intermediate (in time) between Obs. I1 and Obs. I2. 